'Sección Medicina Nuclear, Departamento de Medicina, Hospital Clínico Universidad de Chile.

2Departamento de Cardiología y Cirugía Cardiovascular, Hospital Clínico Universidad de Chile.

${ }^{3}$ Centro de Apoyo a la Investigación Científica OAIC, Hospital Clínico Universidad de

Chile.

Correspondencia a:

Dra. Teresa Massardo

Sección Medicina Nuclear Hospital Clínico Universidad de Chile

Teléfono: +56229788184 tmassardo@hcuch.cl

\section{Seguimiento clínico en pacientes con buena capacidad metabólica en la prueba de esfuerzo con SPECT miocárdico}

\author{
JAVIERA GONZÁLEZ ${ }^{1}$, HERNÁN PRAT ${ }^{2}$, EDUARDO SWETT ${ }^{1}$, \\ ISABEL BERROCAL ${ }^{1}$, RENÉ FERNÁNDEZ ${ }^{1}$, JUAN PABLO ZHINDON ${ }^{1}$, \\ ARIEL CASTRO $^{3}$, TERESA MASSARDO ${ }^{1}$
}

\section{Follow-up of patients with good exercise capacity in stress test with myocardial single-photon emission computed tomography (SPECT)}

Background: The evaluation of coronary artery disease (CAD) can be performed with stress test and myocardial SPECT tomography. Aim: To assess the predictive value of myocardial SPECT using stress test for cardiovascular events in patients with good exercise capacity. Material and Methods: We included 102 males aged $56 \pm 10$ years and 19 females aged $52 \pm 10$ years, all able to achieve 10 METs and $\geq 85 \%$ of the theoretical maximum heart rate and at least $8 \mathrm{~min}$ in their stress test with gated $99 \mathrm{mTc}$-sestamibi SPECT. Eighty two percent of patients were followed clinically for $33 \pm 17$ months. Results: Sixty seven percent of patients were studied for CAD screening and the rest for known disease assessment. Treadmill stress test was negative in $75.4 \% ; 37 \%$ of patients with moderate to severe Duke Score presented ischemia. Normal myocardial perfusion SPECT was observed in $70.2 \%$. Reversible defects appeared in $24.8 \%$ of cases, which were of moderate or severe degree ( $>10 \%$ left ventricular extension) in 56.6\%. Only seven cases had coronary events after the SPECT. Two major (myocardial infarction and emergency coronary revascularization) and 5 minor events (elective revascularization) ere observed in the follow-up. In a multivariate analysis, SPECT ischemia was the only statistically significant parameter that increased the probability of having a major or minor event. Conclusions: Nearly a quarter of our patients with good exercise capacity demonstrated reversible defects in their myocardial perfusion SPECT. In the intermediate-term follow-up, a low rate of cardiac events was observed, being the isotopic ischemia the only significant predictive parameter.

(Rev Med Chile 2015; 143: 1426-1434)

Key words: Coronary artery disease; Exercise test; Technetium Tc $99 \mathrm{~m}$ Sestamibi; Tomography, emission-computed, single photon.
E 1 desempeño durante la prueba de esfuerzo (PE) proporciona información clínica en la evaluación de enfermedad coronaria $(\mathrm{EC})^{1,2}$. Un número alto de equivalentes metabólicos (METs) tiene valor predictivo negativo para eventos cardiacos, independiente de la condición cardiovascular aunque pudiera subestimarlos ${ }^{3,4}$.

Un índice propuesto por investigadores de la Universidad de Duke con valor pronóstico en EC $^{5,6}$ incluye parámetros de la PE [duración, en 
min-(5 x la máxima desviación del segmento ST durante o post-ejercicio, en milímetros)-( 4 x índice de angina)]. El estrés farmacológico se asocia a menor sobrevida en todos los subgrupos de edad, sin considerar los factores de riesgo cardiovascular $(\mathrm{FRCV})^{7}$.

La tomografía computada de emisión de fotón único (SPECT) es superior a la PE para detección de $\mathrm{EC}^{8}$, con buen valor predictivo negativo; así un estudio normal tiene baja tasa de eventos mayores, en seguimiento a largo plazo $^{7}$; con ejercicio, en pacientes sin EC conocida, presenta tasa de mortalidad $<1 \%$ anual, desde $0,2 \%$ anual sin FRCV y hasta $1,7 \%$ anual con tres de ellos. Un gran estudio que midió su impacto sobre muerte, infarto de miocardio (IM) y revascularización coronaria demostró que tiene implicancia pronóstica con cualquier nivel de ejercicio y que aunque los defectos isquémicos fueron poco frecuentes sobre 10 METs estuvieron fuertemente asociados a eventos cardíacos $^{10}$.

Por otra parte, la población diabética es de mayor riesgo cardiovascular, debido a que puede ser asintomática; en ellos las pruebas de isquemia poseen valor predictivo negativo a menor plazo que en la población general; dependiendo de factores como status clínico, función sistólica izquierda post-estrés y capacidad metabólica ${ }^{11,12}$; en un proyecto con diabéticos asintomáticos sin EC conocida, se demostró isquemia en $25 \%$ de los $\operatorname{casos}^{13}$. Se ha planteado tomar en cuenta más variables que los METs para estratificar riesgo en esta población ${ }^{14}$. En EC conocida o diabetes mellitus un SPECT de perfusión normal con estrés posee buen valor pronóstico, incluso con dilatación isquémica transitoria del ventrículo izquierdo ${ }^{15}$.

En la actualidad, se discuten las indicaciones para realizar imágenes de perfusión miocárdica en población con probabilidad intermedia de EC. En trabajos que dividen la población según METs alcanzados y frecuencia cardiaca máxima teórica (FCMT), el porcentaje de eventos a corto o largo plazo en pacientes con alta capacidad metabólica sería mínimo y no afectaría la sobrevida ${ }^{16,17}$. Con la implementación de los criterios apropiados para imágenes cardiacas se intenta acotar costos, aumentar seguridad y disminuir radiación, seleccionando mejor la población con mayor riesgo de $\mathrm{EC}^{18-20}$.

Por lo tanto, nuestro objetivo fue conocer el valor pronóstico de la isquemia en SPECT mio- cárdico para eventos cardiovasculares en pacientes con buena capacidad metabólica asociado a índice de $D u k e$, en población referida a nuestro centro.

\section{Materiales y Métodos}

Se revisaron 2.571 informes consecutivos de SPECT miocárdico entre 2005-2011, de éstos, 780 pacientes efectuaron PE; se incluyeron 121 casos con $\geq 10$ METs, más de $85 \%$ de la FCMT y al menos 8 min de duración de la PE en plataforma. El seguimiento fue vía telefónica, ficha clínica y consulta a registro civil para evaluar mortalidad por todas las causas. El estudio contó con aprobación del Comité de Ética Científico.

\section{PE e imágenes SPECT}

Los pacientes ejecutaron protocolo Bruce. Se consideró electrocardiograma positivo a la depresión del ST de al menos $1 \mathrm{~mm}$ horizontal o descendente 80 milisegundos después del punto $\mathrm{J}$ en tres ciclos consecutivos. La PE fue positiva si estaba eléctricamente alterada y/o presentaba angina típica. Para el análisis, los electrocardiogramas no concluyentes fueron considerados negativos.

El SPECT gatillado fue efectuado con $\mathrm{Tc}^{99 \mathrm{~m}_{-}}$ sestamibi en reposo y esfuerzo; con actividad entre 370 y $1110 \mathrm{MBq}$ usando cámara Siemens E. Cam Dual head. Se utilizó programa Cedars Sinai $Q P S^{\circledast}$ y $Q G S^{\circledast}$ obteniendo volúmenes y fracción de eyección ventricular izquierda (FEVI), índices sumados de estrés (SSS), de reposo (SRS), diferencial (SDS) y déficit de perfusión total (TPD). Se definió isquemia significativa $\geq 10 \%$ de compromiso del ventrículo izquierdo; la interpretación utilizó análisis cualitativo y cuantitativo según extensión y SDS.

Los eventos mayores correspondieron a muerte de causa coronaria e IM no fatal. Los menores a angina inestable y revascularización coronaria electiva.

\section{Análisis estadístico}

Prueba de $t$-student o Mann Whitney según normalidad de los datos para comparar variables continuas y $\chi^{2}$ o Fisher para categóricas ( $\mathrm{p}$ significativa $<0,05)$. Se efectuó análisis bi y multivariado de sobrevida libre de eventos con riesgo proporcional de Cox para parámetros clínicos, electrocardiográficos y de SPECT (en análisis multivariado $\mathrm{p}<0,2)$ con $S T A T A^{\oplus} \mathrm{v} 12.1$. 


\section{Resultados}

\section{Generales}

Las características del grupo se muestran en Tabla 1. El SPECT fue solicitado para detección de EC en $67 \%$ de los casos y para evaluar enfermedad conocida en el resto (IM en 11,6\% y/o revascularización miocárdica en $27 \%, 73 \%$ percutánea). Un $46 \%$ del total refería dolor torácico previo. Los estudios preoperatorios de cirugía no cardiaca correspondían a $7 \%$ en hombres y $16 \%$ en mujeres $(p=n s)$. El índice de masa corporal en hombres varió entre 22 y $37 \mathrm{~kg} / \mathrm{m}^{2}$ y en mujeres entre 17 y $43 \mathrm{~kg} / \mathrm{m}^{2}$. No hubo casos de valvulopatías, miocardiopatías, marcapasos, insuficiencia renal ni consumo de sustancias. El SPECT fue solicitado en $86 \%$ por cardiólogos.

\section{Prueba de esfuerzo}

Los pacientes realizaron protocolo de Bruce, todos con capacidad funcional NYHA I. El electrocardiograma basal fue normal o cercano a esto en $75 \%$, con onda Q en $10,5 \%$ y otros trastornos de conducción en 14,5\%. Sus parámetros promedio fueron: duración 9,8 \pm 1,3 min; FCMT 94,6 \pm 7\%; METs 11,8 $\pm 1,6$ y motivos de suspensión: alcanzar FCMT (53\%), fatiga (46\%) y el resto angina, disnea, $y /$ o respuesta hipertensiva inapropiada. $\mathrm{La}$ PE fue positiva en $26,4 \%$.

El índice de Duke fue leve (bajo riesgo) en $72 \%$ del total de pacientes, moderado en $23 \%$ (intermedio) y severo en 5\% (alto). El 37\% de los casos con índice moderado o severo presentaron isquemia en SPECT.

Tabla 1. Principales características clínicas, hallazgos en prueba de esfuerzo y en SPECT en la muestra estudiada (promedio \pm desviación estándar)

\begin{tabular}{|c|c|c|c|c|}
\hline & & Hombres & Mujeres & $\mathbf{p}$ \\
\hline \multicolumn{2}{|l|}{ Casos } & $102(84,3 \%)$ & $19(15,7 \%)$ & \\
\hline \multicolumn{2}{|l|}{ Años de edad } & $56,5 \pm 10,3$ & $51,6 \pm 10,3$ & 0,029 \\
\hline \multicolumn{2}{|l|}{ Peso (kg) } & $80,1 \pm 11,7$ & $66,3 \pm 15,6$ & 0,0005 \\
\hline \multicolumn{2}{|c|}{ Índice masa corporal $\left(\mathrm{kg} / \mathrm{m}^{2}\right)$} & $27,7 \pm 3,3$ & $27,5 \pm 5,8$ & ns \\
\hline \multicolumn{2}{|l|}{ EC conocida } & $40,1 \%$ & $0 \%$ & 0,0003 \\
\hline FRCV & $\begin{array}{l}\text { Hipertensión arterial } \\
\text { Diabetes } \\
\text { Tabaquismo } \\
\text { Dislipidemia }\end{array}$ & $\begin{array}{l}68,4 \% \\
26,8 \% \\
31,3 \% \\
44,4 \%\end{array}$ & $\begin{array}{c}31,6 \% \\
5,3 \% \\
35,7 \% \\
40,4 \%\end{array}$ & $\begin{array}{l}0,002 \\
0,032 \\
\text { ns } \\
\text { ns }\end{array}$ \\
\hline Prueba esfuerzo & $\begin{array}{l}\text { Minutos } \\
\text { FCMT } \\
\text { Doble Producto } \\
\text { METs } \\
\text { Positiva } \\
\text { Negativa/no concluyente } \\
\text { Leve }(\geq 5)\end{array}$ & $\begin{array}{c}9,9 \pm 1,4 \\
94,9 \pm 6,9 \% \\
28,224 \pm 3,162 \\
11,8 \pm 1,7 \\
31(31,4 \%) \\
70(68,6 \%) \\
67 \%\end{array}$ & $\begin{array}{c}9,1 \pm 0,9 \\
93,3 \pm 4,9 \% \\
27,522 \pm 3,061 \\
10,6 \pm 1,2 \\
1(5,3 \%) \\
18(94,7 \%) \\
84 \%\end{array}$ & $\begin{array}{c}<0,0001 \\
\text { ns } \\
\text { ns } \\
0,048 \\
0,013\end{array}$ \\
\hline Índice de Duke & $\begin{array}{l}\text { Moderado }(5 \mathrm{y}<11) \\
\text { Severo }(\geq 11)\end{array}$ & $\begin{array}{c}24 \% \\
6 \%\end{array}$ & $\begin{array}{c}16 \% \\
0 \%\end{array}$ & ns \\
\hline Isquemia en SPECT & $\begin{array}{l}\text { Positivo } \\
\text { Negativo }\end{array}$ & $\begin{array}{l}28(27,4 \%) \\
74(72,5 \%)\end{array}$ & $\begin{array}{c}2(10,5 \%) \\
17(89,5 \%)\end{array}$ & ns \\
\hline FEVI & $\begin{array}{l}\text { Post-esfuerzo } \\
\text { Reposo }\end{array}$ & $\begin{array}{l}63,7 \pm 12,0 \\
63,1 \pm 11,3\end{array}$ & $\begin{array}{l}70,5 \pm 16,3 \\
69,3 \pm 15,4\end{array}$ & $\begin{array}{l}0,018 \\
0,022\end{array}$ \\
\hline Eventos & $\begin{array}{l}\text { Mayores } \\
\text { Menores }\end{array}$ & $\begin{array}{l}1 \\
5\end{array}$ & $\begin{array}{l}1 \\
0\end{array}$ & \\
\hline
\end{tabular}

FEVI: Fracción de eyección del ventrículo izquierdo (\%). FCMT: Frecuencia cardíaca máxima teórica (latidos/min). METs: equivalentes metabólicos $\left(\mathrm{kcal} \cdot \mathrm{kg}^{-1} \cdot \mathrm{h}^{-1}\right)$. Doble producto: Frecuencia cardíaca (latidos/minutos) x presión arterial sistólica máximas $(\mathrm{mm} \mathrm{Hg})$. 


\section{SPECT gatillado}

El examen de perfusión fue normal en $70,2 \%$. Entre los anormales se encontraron $24,8 \%$ de defectos reversibles siendo $56,6 \%$ de ellos isquemia significativa (moderada o severa). Los parámetros cuantitativos de los casos isquémicos fueron: $\mathrm{SSS}=12 \pm 6$; $\mathrm{SDS}=6 \pm 4,8$; $\mathrm{TPD}$ estrés $=14 \pm 6$. Además, en $43 \%$ de los defectos reversibles hubo isquemia con algún componente fijo. El 5\% del total de pacientes tuvo defectos no reversibles, todos ellos tenían EC conocida y 4/6 IM previo.

Los pacientes presentaron cavidad ventricular izquierda de tamaño y función adecuada, salvo uno dilatado y dos con FEVI de 36 y $32 \%$ en reposo con hipokinesia, explicable por IM.

Existió asociación significativa entre presencia de isquemia en SPECT e índice de Duke (Figura 1).

\section{Seguimiento}

El seguimiento completo se logró en 82,3\% de 121 casos (promedio $33 \pm 17$ meses, rango 14-82). No hubo muertes de origen cardíaco o accidentes cerebrovasculares. Sólo 7 casos presentaron eventos coronarios, 2 mayores que correspondieron a síndrome coronario agudo, uno requirió 4 puentes coronarios y otro revascularización percutánea de urgencia; además, 5 eventos menores en pacientes con revascularización electiva post-coronariografía que se efectuó basada en antecedentes clínicos, resultados del SPECT cuando fue positivo y en presencia de le-

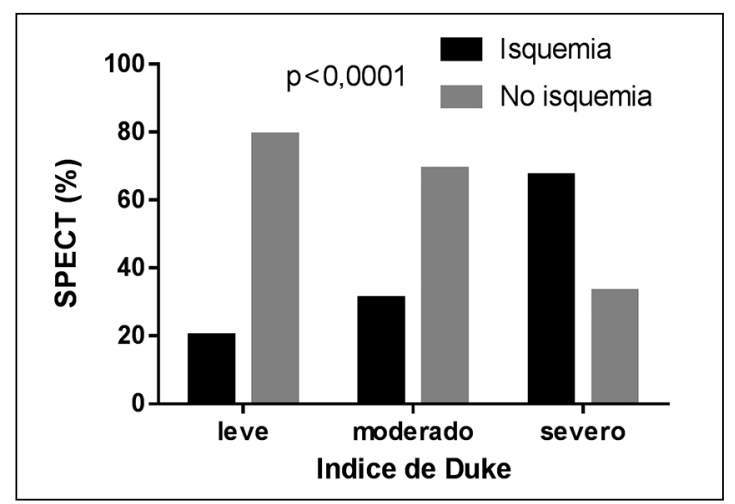

Figura 1. Análisis porcentual de presencia o ausencia de isquemia en SPECT miocárdico e índice de Duke según grado en la PE; prueba de $\chi^{2}$. siones significativas. En dos casos hubo aparición de disnea (en uno progresiva). No hubo otros antecedentes de que estos pacientes presentaran nuevos síntomas ni otros estudios angiográficos coronarios (Figuras 2 y 3 ).

El seguimiento indirecto adicional incluyendo a los pacientes sin contacto clínico tuvo promedio de $50 \pm 18$ meses (rango: 25-106) en el que no se constataron fallecimientos de ninguna causa.

Al revisar los resultados de la PE y el SPECT, los casos que presentaron eventos mayores, tenían antecedentes de angor y adecuada indicación del estudio. Uno correspondió a paciente de 59 años con EC conocida, depresión del segmento $\mathrm{ST}$ e isquemia leve en SPECT cuyo evento se presentó a los 147 días del estudio. El otro a hipertenso de 51 años sin lesiones significativas en coronariografía 1 año previo al estudio con evento a los 302 días post SPECT, sin isquemia. En los eventos menores, 4 de 5 tuvieron isquemia en SPECT (moderada o severa en 2 y leve en el resto), su índice de Duke fue moderado o severo. La revascularización coronaria se efectuó entre 13 y 754 días post-SPECT. De los SPECT positivos para isquemia en grado leve que tuvieron revascularización coronaria, todos fueron coronarios conocidos con PE alterada, el primero, diabético asintomático, dislipidémico con angioplastia previa a la arteria descendente anterior y puente coronario a los 4 meses post-SPECT; el segundo, con IM y angioplastia a la arteria circunfleja 3 años previo al estudio, quien recibió 3 puentes el mes siguiente al SPECT; el tercero, un paciente multivaso con cuádruple cirugía de puentes 2 años atrás y doble angioplastia con stent medicado 1 mes posterior a nuestro estudio.

Con respecto a la sobrevida a 5 años libre de eventos cardiovasculares, en análisis bivariado, se encontraron asociaciones significativas con presencia de isquemia en SPECT con Hazard Ratio (HR) de 13,59; ( $\mathrm{p}=0,02)$, índice de Duke moderado o severo (HR: 11,$44 ; \mathrm{p}=0,029)$ y PE positiva (HR: 10,19; $\mathrm{p}=0,036$ ). En análisis multivariado, la isquemia en SPECT fue estadísticamente significativa (HR: 9,52; $\mathrm{p}=0,046)$ y la PE positiva no alcanzó significancia (HR: 8,12 ; $p=0,064)$. Ambos análisis no fueron significativos para eventos mayores.

En los resultados de sobrevida libre de eventos compuestos hubo asociaciones significativas para 


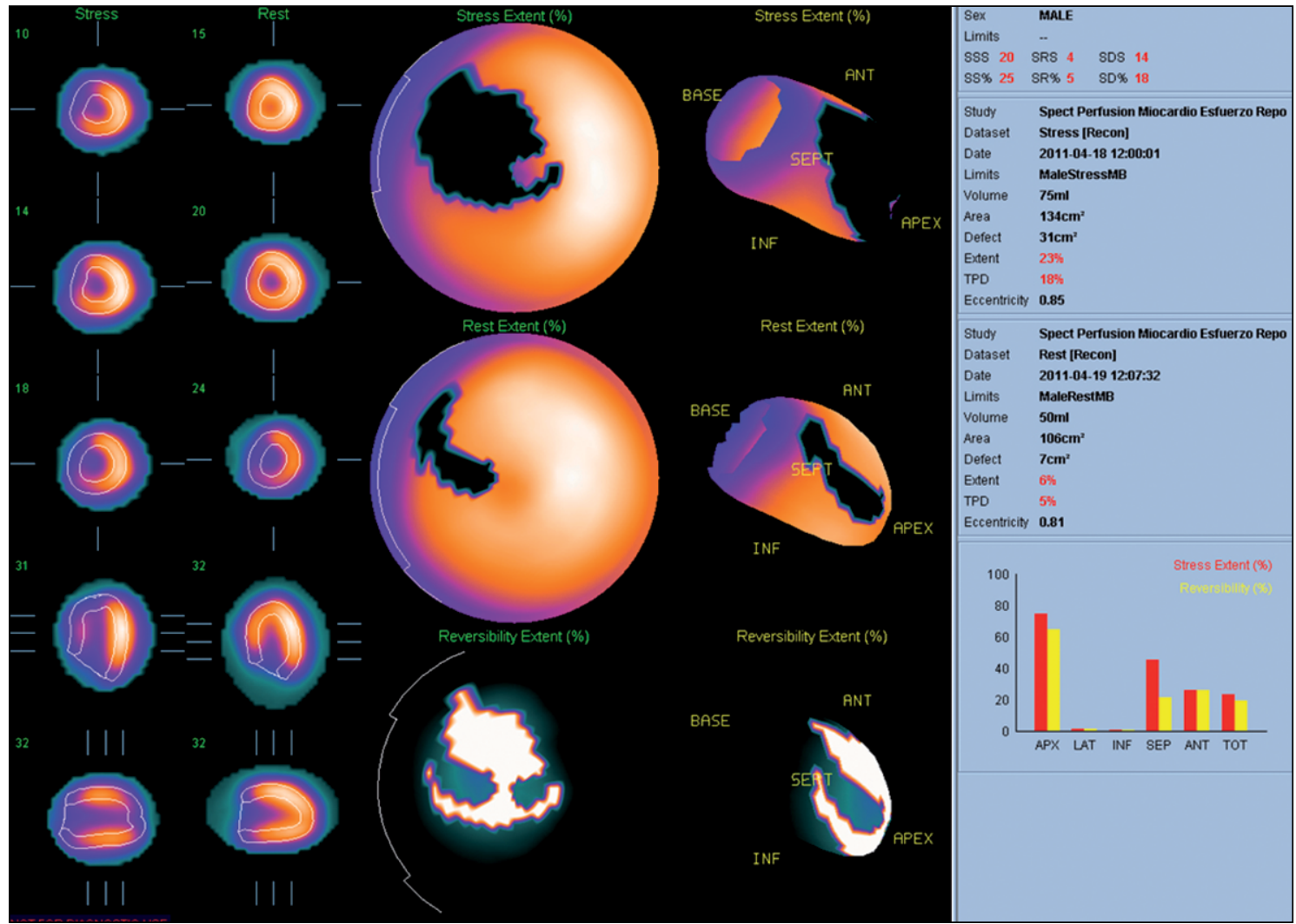

Figura 2. Paciente de sexo masculino de 52 años derivado para estudio de EC por angina. Realiza prueba de Bruce, $10 \mathrm{METs}$, 8,4 min, 89\% de FCMT e índice de Duke con riesgo moderado. SPECT con severa isquemia anteroseptoapical. SDS: 14; extensión en estrés: 23\%. FEVI normal. La angiografía coronaria electiva (evento menor) 13 días post SPECT concluyó enfermedad de 3 vasos. Terapia: puente de mamaria interna a arteria coronaria descendente anterior, sin complicaciones posteriores.

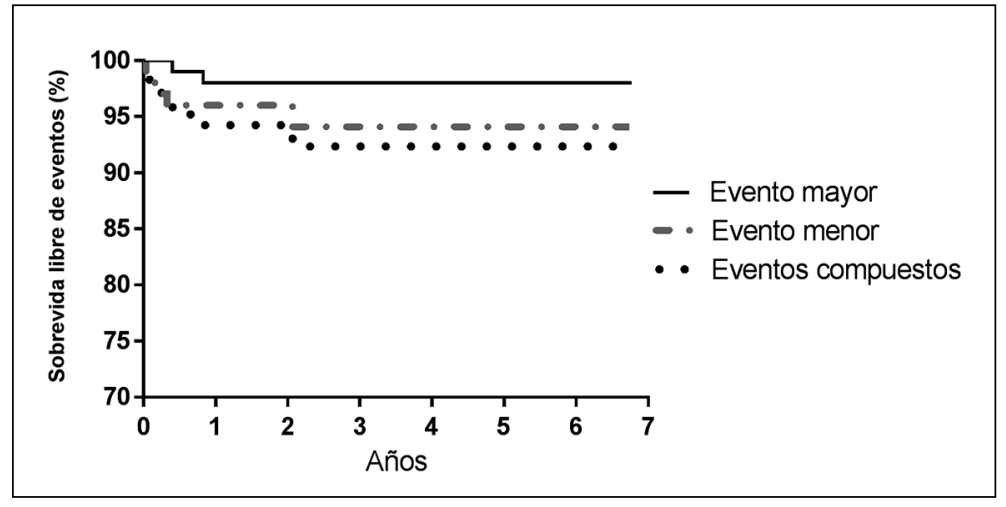

Figura 3. Resultados de sobrevida libre de eventos mayores, menores y compuestos en el tiempo de seguimiento en todos los pacientes. isquemia en SPECT (HR: 6,23; $\mathrm{p}=0,001)$ e índice de Duke moderado o severo (HR: 7,15; $\mathrm{p}=0,019$ ). En análisis bivariado, solamente para isquemia en SPECT (HR: 6,87; $\mathrm{p}=0,022$ ). El análisis multi- variado de eventos compuestos, fue significativo para isquemia en SPECT (HR: 6,66; $\mathrm{p}=0,028$ ) y sólo tuvo tendencia la PE positiva (HR: 4,24; $\mathrm{p}=0,09)$ (Tabla 2). 
Tabla 2. Análisis bivariado y multivariado de FRCV e isquemia en PE y SPECT expresados como Hazard Ratio

\begin{tabular}{|c|c|c|c|c|c|}
\hline & & & $\begin{array}{l}\text { Eventos } \\
\text { menores }\end{array}$ & $\begin{array}{l}\text { Eventos } \\
\text { mayores }\end{array}$ & $\begin{array}{c}\text { Eventos } \\
\text { compuestos }\end{array}$ \\
\hline Análisis bivariado & $\begin{array}{l}\text { FRCV } \\
\text { PE po } \\
\text { Score } \\
\text { Isquer } \\
\text { Dolor } \\
\text { Preser }\end{array}$ & $\begin{array}{l}\text { HTA } \\
\text { Diabetes } \\
\text { Tabaquismo } \\
\text { Dislipidemia } \\
\text { Dderado a severo } \\
\text { T } \\
\text { orevio }\end{array}$ & $\begin{array}{c}* * \\
0,76(0,08-7) \\
0,88(0,09-8) \\
0,83(0,14-5) \\
10,49(1,17-94)^{*} \\
11,44(1,28-103)^{*} \\
13,59(1,52-122)^{*} \\
0,32(0,04-3) \\
3,15(0,52-19)\end{array}$ & $\begin{array}{c}* * \\
* * \\
* * \\
1,32(0,82-21) \\
2,49(0,16-40) \\
2,60(0,16-42) \\
3,23(0,20-52) \\
* * \\
* *\end{array}$ & $\begin{array}{l}\quad * * \\
0,56(0,07-5) \\
0,68(0,07-7) \\
0,96(0,21-4) \\
6,23(1,21-32)^{*} \\
7,14(1,38-37) \\
9,11(1,72-48)^{*} \\
0,94(0,21-4) \\
1,52(0,34-7)\end{array}$ \\
\hline Análisis multivariado & $\begin{array}{l}\text { PE po } \\
\text { Isquer }\end{array}$ & & $\begin{array}{l}8,13(0,88-75) \\
9,52(1,04-87)^{*}\end{array}$ & $\begin{array}{l}* * \\
* *\end{array}$ & $\begin{array}{l}4,24(0,79-23) \\
6,66(1,23-36)^{*}\end{array}$ \\
\hline
\end{tabular}

${ }^{*} \mathrm{p}<0,05 .{ }^{* *}$ No calculable, debido a tamaño muestral reducido o número reducido de eventos.

\section{Discusión}

Evitar pruebas de estrés farmacológico, efectuando PE en pacientes aptos para ello, aporta parámetros electrocardiográficos independientes, logra objetivar la angina y la capacidad funcional. Por lo demás, en población femenina, existe discusión respecto a la indicación del SPECT con ejercicio como evaluación inicial en sintomáticas con sospecha de EC, intentando estudiar sólo población intermedia-alta ${ }^{21}$ disminuyendo falsos positivos. En general, se sobreutiliza el estrés farmacológico como provocador de isquemia, que corresponde a $60 \%$ del total de SPECT en estrés en nuestro centro.

Un estudio realizado en un gran grupo de pacientes sin EC con ecocardiografía de ejercicio y buena capacidad metabólica ( $\geq 85 \%$ FCMT y $\geq 10$ METs) encontró isquemia en $14 \%$ (extensa en $9,3 \%$ ) y $6 \%$ de enfermedad multiterritorio, con tasa de mortalidad anual de $1 \%$ y eventos cardíacos mayores de $0,4 \%$, valores menores que los observados en pacientes que no alcanzaron esa carga. Las bajas tasas observadas parecieran limitar la utilidad de la técnica para evaluar eventos ${ }^{22}$. Aunque nuestra casuística es limitada por el tipo de pacientes, la tasa de eventos mayores fue más alta $(1,7 \%)$ que la descrita ${ }^{16}$. En otro estudio con $\mathrm{Tl}^{201}$-SPECT en pacientes sin EC e índices de Duke alto y bajo, con seguimiento a 7 años, se observó que sólo en alto riesgo el SPECT tuvo valor pronóstico independiente para mortalidad cardiaca ${ }^{23}$. Sin embargo, la experiencia reciente en pacientes con edad marcadamente superior a la nuestra, de los cuales 28\% realizó PE, el alcanzar $<7$ METs y el aumento del volumen de fin de sístole predijeron mortalidad; las variables del SPECT fueron incapaces de proveer estratificación del riesgo ${ }^{24}$.

Otro predictor de mortalidad reconocido es el retardo de la respuesta cronotrópica al ejercicio, incluso en casos con respuesta electrocardiográfica normal, probablemente superior al índice de Duke descrito también como factor independiente de eventos cardiovasculares en diabéticos ${ }^{25,26}$; nuestros diabéticos no tuvieron mayor tasa de eventos.

En el trabajo presentado destaca que entre los SPECT con esfuerzo, la proporción de pacientes con excelente capacidad de ejercicio fue relativamente alta $(30,3 \%)$. La muestra correspondió a casos derivados para evaluación de EC; aproximadamente $25 \%$ presentaba isquemia en las imágenes, significativa en más de la mitad, sin diferencias entre los con y sin antecedente de EC; el índice de Duke también fue similar y en el seguimiento, ambos grupos tuvieron algún evento menor. De ellos, casi la mitad eran sintomáticos al momento del estudio; 5 de los 7 pacientes con eventos tuvieron isquemia en SPECT, significativa sólo en 2. La ausencia de mortalidad de origen cardiovascular y la baja tasa de IM agudo (2\%) dentro del año posterior a la realización del SPECT en pacientes con alto rendimiento aeróbico fue concordante con la literatura ${ }^{12,16,17,27}$. Otro estudio reciente le asigna valor predictivo en la $\mathrm{PE}$ a la tasa del doble producto ${ }^{28}$. No le encontramos 
valor pronóstico a esta variable, explicable por la selección de la muestra.

$\mathrm{Al}$ hacer análisis por género, nuestras mujeres eran más jóvenes, tenían menos EC conocida, diabetes mellitus e hipertensión arterial, presentaban mayor FEVI en post-esfuerzo, lo cual podría ser explicable por sobreestimación del volumen de cavidades pequeñas por software.

En este análisis, la presencia de isquemia en el SPECT miocárdico y el índice de Duke moderado a severo tuvieron valor pronóstico de eventos coronarios menores y compuestos. Además, la isquemia evaluada por método isotópico fue el único parámetro que aumentó significativamente la probabilidad de presentar un evento compuesto en análisis multivariado expresado como Hazard Ratio. Ni el número de FRCV, ni la PE, ni el índice de Duke alcanzaron significancia para establecer riesgo de eventos. Tampoco hubo resultados significativos al analizar los eventos mayores, hallazgo explicable por la baja incidencia de estos. Debiera considerarse la probabilidad de EC previa y el número de FRCV, pues en un trabajo con SPECT miocárdicos negativos con 6 años de seguimiento, la mortalidad a largo plazo aumentó significativamente según el número de factores ${ }^{9,29}$.

Las guías actuales para evaluación de EC estable aconsejan pruebas de detección de isquemia con imágenes en pacientes con probabilidad pre-test de EC entre $65-85 \%$, FEVI > 50\% sin angina típica $\mathrm{o}$ alteraciones del electrocardiograma basal ${ }^{30,31}$.

\section{Fortalezas y limitaciones}

En este trabajo es destacable que se incluyó a una población de pacientes de la práctica clínica de un hospital universitario, con buena capacidad metabólica, consecutivos, con riesgo intermedio de EC, casi la mitad sintomáticos, con seguimiento a mediano plazo. Una fortaleza importante de la técnica es su alto valor predictivo negativo, que aunque no fue cuantificada en forma directa, está avalada por la baja incidencia de eventos, esperable en este grupo con alta capacidad aeróbica. Hubiera sido interesante contar con estudio prospectivo, mayor seguimiento, información clínica y casuística. Es probable haber incluido cierto sesgo al considerar la revascularización electiva como evento cardiaco menor en pacientes derivados a estudio de perfusión. La sensibilidad del SPECT de miocardio es alrededor de $85 \%$ lo que explica el resultado falso negativo de los pacientes con evento mayor y menor que no demostraron isquemia y que pudiera deberse en parte a enfermedad de microvaso o alteración muy pequeña no visualizada por problema técnico.

\section{Conclusiones}

Nuestros pacientes con buena capacidad de ejercicio con riesgo intermedio de EC (promedio 1,8 FRCV), algunos con EC conocida, presentaron defectos de tipo isquémico con SPECT en 25\%, de los cuales sobre $50 \%$ fue significativo. En seguimiento a mediano plazo no hubo mortalidad cardiovascular, se observó baja tasa de eventos cardíacos mayores ( $<1 \%$ anual). Un $17 \%$ de aquellos con isquemia isotópica fueron sometidos a revascularización. Es importante destacar que isquemia en SPECT miocárdico y el índice de Duke moderado o severo son factores pronósticos independientes de eventos coronarios menores y compuestos.

\section{Referencias}

1. Wackers FJ. Customized Exercise Testing. J Am Coll Cardiol 2009; 54 (6): 546-8.

2. Shaw LJ, Peterson ED, Shaw LK, Kesler KL, DeLong ER, Harrell FE Jr. et al. Use of a prognostic treadmill score in identifying diagnostic coronary disease subgroups. Circulation 1998 (16); 98: 1622-30.

3. Peterson PN, Magid DJ, Ross C, Ho PM, Rumsfeld JS, Lauer MS, et al. Association of exercise capacity on treadmill with future cardiac events in patients referred for exercise testing. Arch Intern Med 2008; 168 (2): 174-9.

4. Pinkstaff S, Peberdy MA, Kontos MC, Fabiato A, Finucane S, Arena R. Overestimation of aerobic capacity with the Bruce treadmill protocol in patients being assessed for suspected myocardial ischemia. J Cardiopulm Rehabil Prev 2011; 31 (4): 254-60.

5. Mark DB, Shaw L, Harrell FE Jr, Hlatky MA, Lee KL, Bengtson JR, et al. Prognostic value of a treadmill exercise score in outpatients with suspected coronary artery disease. N Engl J Med 1991; 325 (12): 849-53.

6. Lakkireddy DR, Bhakkad J, Korlakunta HL, Ryschon K, Shen X, Mooss AN, et al. Prognostic value of the Duke Treadmill Score in diabetic patients. Am Heart J 2005; 150 (3): 516-21. 
7. Uretsky S, Rozanski A. Long-term outcomes following a normal stress myocardial perfusion scan. J Nucl Cardiol 2013; 20 (5): 715-8.

8. Hachamovitch R, Berman DS, Kiat H, Cohen I, Friedman JD, Shaw LJ. Value of stress myocardial perfusion single photon emission computed tomography in patients with normal resting electrocardiograms: an evaluation of incremental prognostic value and cost-effectiveness. Circulation 2002; 105 (7): 823-9.

9. Supariwala A, Uretsky S, Singh P, Memon S, Khokhar SS, Wever-Pinzon O, et al. Synergistic effect of coronary artery disease risk factors on long-term survival in patients with normal exercise SPECT studies. J Nucl Cardiol 2011; 18 (2): 207-14.

10. Lee DS, Verocai F, Husain M, Al Khdair D, Wang X, Freeman M. et al. Cardiovascular outcomes are predicted by exercise-stress myocardial perfusion imaging: Impact on death, myocardial infarction, and coronary revascularization procedures. Am Heart J 2011; 161 (5): 900-7.

11. Acampa W, Petretta M, Cuocolo R, Daniele S, Cantoni $\mathrm{V}$, Cuocolo A. Warranty period of normal stress myocardial perfusion imaging in diabetic patients: a propensity score analysis. J Nucl Cardiol 2014; 21 (1): 50-6.

12. Padala SK, Ghatak A, Padala S, Katten DM, Polk DM, Heller GV. Cardiovascular risk stratification in diabetic patients following stress single-photon emission-computed tomography myocardial perfusion imaging: The impact of achieved exercise level. J Nucl Cardiol 2014; 21 (6): 1132-43.

13. Hage G, Lusa L, Dondi M, Giubbini R, Iskandrian AE; IAEA Diabetes Investigators. Exercise stress tests for detecting myocardial ischemia in asymptomatic patients with diabetes mellitus. Am J Cardiol 2013; 112 (1): 1420.

14. Petretta M, Acampa W, Cuocolo A. Cardiovascular risk stratification in diabetic patients: Is all in METS? J Nucl Cardiol 2014; 21 (6): 1144-7.

15. Doukky R, Hayes K, Frogge N, Balakrishnan G, Dontaraju VS, Rangel MO, et al. Impact of appropriate use on the prognostic value of single-photon emission computed tomography myocardial perfusion imaging. Circulation 2013; 128 (15): 1634-43.

16. Bourque JM, Holland BH, Watson DD, Beller GA. Achieving an exercise workload of $>$ or $=10$ metabolic equivalents predicts a very low risk of inducible ischemia: does myocardial perfusion imaging have a role? J Am Coll Cardiol 2009; 54 (6): 538-45.

17. Bourque JM, Charlton GT, Holland BH, Belyea CM, Watson DD, Beller GA. et al. Prognosis in patients achieving $\geq 10$ METS on exercise stress testing: was SPECT imaging useful? J Nucl Cardiol 2011; 18 (2): 230-7.

18. Beller GA Bateman TM. Provisional use of myocardial perfusion imaging in patients undergoing exercise stress testing: A worthy concept fraught with challenges. J Nucl Cardiol 2013; 20 (5): 711-4.

19. Wolk MJ, Bailey SR, Doherty JU, Douglas PS, Hendel RC, Kramer CM, et al. American College of Cardiology Foundation Appropriate Use Criteria Task Force.ACCF/ AHA/ASE/ASNC/HFSA/HRS/SCAI/SCCT/SCMR/ STS 2013 multimodality appropriate use criteria for the detection and risk assessment of stable ischemic heart disease: a report of the American College of Cardiology Foundation Appropriate Use Criteria Task Force, American Heart Association, American Society of Echocardiography, American Society of Nuclear Cardiology, Heart Failure Society of America, Heart Rhythm Society, Society for Cardiovascular Angiography and Interventions, Society of Cardiovascular Computed Tomography, Society for Cardiovascular Magnetic Resonance, and Society of Thoracic Surgeons. J Am Coll Cardiol 2014; 63 (4): 380-406.

20. Doukky R, Frogge N, Bayissa YA, Balakrishnan G, Skelton JM, Confer K, et al. The prognostic value of transient ischemic dilatation with otherwise normal SPECT myocardial perfusion imaging: a cautionary note in patients with diabetes and coronary artery disease. J Nucl Cardiol 2013; 20 (5): 774-84.

21. Shaw LJ, Mieres JH, Hendel RH, Boden WE, Gulati M, Veledar E, et al; WOMEN Trial Investigators. Comparative effectiveness of exercise electrocardiography with or without myocardial perfusion single photon emission computed tomography in women with suspected coronary artery disease: results from the What Is the Optimal Method for Ischemia Evaluation in Women (WOMEN) trial. Circulation 2011; 124 (11): 1239-49.

22. Peteiro J, Bouzas-Mosquera A, Broullón F, Martínez D, Yáñez J, Castro-Beiras A. Value of an exercise workload $\geq 10$ metabolic equivalents for predicting inducible myocardial ischemia. Circ Cardiovasc Imaging 2013; 6 (6): 899-907.

23. Poornima IG, Miller TD, Christian TF, Hodge DO, Bailey KR, Gibbons RJ. Utility of myocardial perfusion imaging in patients with low-risk treadmill scores. J Am Coll Cardiol 2004; 43 (2): 194-9.

24. Kwon DH, Menon V, Houghtaling P, Lieber E, Brunken $\mathrm{RC}$, Cerqueira MD, et al. Predictive value of exercise myocardial perfusion imaging in the Medicare population: the impact of the ability to exercise. Cardiovasc Diagn Ther 2014; 4 (1): 5-12. 
25. Ho PM, Maddox TM, Ross C, Rumsfeld JS, Magid DJ. Impaired chronotropic response to exercise stress testing in patients with diabetes predicts future cardiovascular events. Diabetes Care 2008; 31 (8): 1531-3.

26. Rich JD, Chen S, Ward RP. Comparison of high risk stress myocardial perfusion imaging findings in men with rapid versus prolonged recovery of ST-segment depression after exercise stress testing. Am J Cardiol 2010; 105 (10): 1361-4.

27. Bouzas-Mosquera A, Peteiro J, Álvarez-García N. Value of a high exercise workload to rule out myocardial ischemia. J Am Coll Cardiol 2010; 55 (3): 265-6.

28. Marini C, Acampa W, Bauckneht M, Daniele S, Capitanio S, Cantoni V, et.al. Added prognostic value of ischaemic threshold in radionuclide myocardial perfusion imaging: a common-sense integration of exercise tolerance and ischaemia severity. Eur J Nucl Med Mol Imaging 2015; 42 (5): 750-60.
29. Rozanski A, Gransar H, Min JK, Hayes SW, Friedman JD, Thomson LE, et al. Long-term mortality following normal exercise myocardial perfusion SPECT according to coronary disease risk factors. J Nucl Cardiol 2014; 21 (2): 341-50.

30. Task Force Members, Montalescot G, Sechtem U, Achenbach S, Andreotti F, Arden C, et al. 2013 ESC guidelines on the management of stable coronary artery disease: the Task Force on the management of stable coronary artery disease of the European Society of Cardiology. Eur Heart J 2013; 34 (38): 2949-3003.

31. Working Group of the SEC on the 2013 ESC Guidelines on the Management of Stable Coronary Artery Disease; Reviewers for the 2013 ESC Guidelines on the Management of Stable Coronary Artery Disease; SEC Guidelines Committee. Comments on the 2013 ESC guidelines on the management of stable coronary artery disease. Rev Esp Cardiol 2014; 67 (2): 80-6. 